\title{
Atypical presentation of central pontine myelinolysis in hyperglycemia
}

\author{
Swapna Talluri', Raghu Charumathi' ${ }^{1}$, Muhammad Khan ${ }^{1}$ and Kerri Kissell ${ }^{2}$ \\ IInternal Medicine and 2Endocrinology, Guthrie Robert Packer Hospital, Sayre, Pennsylvania, USA
}

\section{Summary}

Central pontine myelinolysis (CPM) usually occurs with rapid correction of severe chronic hyponatremia. Despite the pronounced fluctuations in serum osmolality, CPM is rarely seen in diabetics. This is a case report of CPM associated with hyperglycemia. A 45-year-old non-smoking and non-alcoholic African American male with past medical history of type 2 diabetes, hypertension, stage $V$ chronic kidney disease and hypothyroidism presented with a two-week history of intermittent episodes of gait imbalance, slurred speech and inappropriate laughter. Physical examination including complete neurological assessment and fundoscopic examination were unremarkable. Laboratory evaluation was significant for serum sodium: $140 \mathrm{mmol} / \mathrm{L}$, potassium: $3.9 \mathrm{mmol} / \mathrm{L}$, serum glucose: $178 \mathrm{mg} / \mathrm{dL}$ and serum osmolality: $317 \mathrm{mosmol} / \mathrm{kg}$. His ambulatory blood sugars fluctuated between 100 and $600 \mathrm{mg} / \mathrm{dL}$ in the six weeks prior to presentation, without any significant or rapid changes in his corrected serum sodium or other electrolyte levels. MRI brain demonstrated a symmetric lesion in the central pons with increased signal intensity on T2- and diffusion-weighted images. After neurological consultation and MRI confirmation, the patient was diagnosed with CPM secondary to hyperosmolar hyperglycemia. Eight-week follow-up with neurology was notable for near-complete resolution of symptoms. This case report highlights the importance of adequate blood glucose control in diabetics. Physicians should be aware of complications like CPM, which can present atypically in diabetics and is only diagnosed in the presence of a high index of clinical suspicion.

\section{Learning points:}

- Despite the pronounced fluctuations in serum osmolality, central pontine myelinolysis (CPM) is rarely seen in diabetics. This case report of CPM associated with hyperglycemia highlights the importance of adequate blood glucose control in diabetics.

- Physicians should be aware of complications like CPM in diabetics.

- CPM can present atypically in diabetics and is only diagnosed in the presence of a high index of clinical suspicion.

\section{Background}

This is a case report of central pontine myelinolysis (CPM) associated with hyperglycemia, which is unique in several aspects:

- CPM occurred in the setting of uncontrolled hyperglycemia with normal corrected serum sodium and no other electrolyte abnormalities.

- The presentation was atypical with intermittent symptoms and patient remained alert without corticospinal involvement.

\section{Case presentation}

A 45-year-old non-smoking and non-alcoholic African American male with past medical history of type 2 diabetes, hypertension, hypothyroidism and stage 5 chronic kidney disease (CKD) presented with a twoweek history of intermittent episodes of gait imbalance, slurred speech and inappropriate laughter. Due to noncompliance with prescribed insulin regimen, he had a history of labile glycemic control. Six weeks prior, he was admitted to the hospital with serum glucose of $600 \mathrm{mg} / \mathrm{dL}$ and corrected serum sodium of

(c) 2017 The authors 
$138 \mathrm{mmol} / \mathrm{L}$ with lack of symptoms, consistent with hyperosmolar hyperglycemia. His physical examination was unremarkable. His management included intravenous fluid resuscitation and intravenous regular insulin $(0.1 \mathrm{U} / \mathrm{kg})$ followed by subcutaneous glargine insulin $(0.25 \mathrm{U} / \mathrm{kg})$. He was observed overnight and was discharged in stable and euglycemic condition the following morning. Following discharge, he continued to be non-compliant with his anti-hyperglycemic regimen as documented by glucometer reports. Four weeks following initial hospitalization, he noticed intermittent gait imbalance. His gait imbalance worsened over time with later development of intermittent slurred speech and episodes of inappropriate laughter lasting for approximately 30 min and occurring multiple times a day. He denied history of head injury, headache, weakness, numbness, visual disturbance, bowel or bladder incontinence and loss of consciousness. His vitals at presentation include temperature: 970F, blood pressure: 168/98, pulse: 93, respiratory rate: 18 , and oxygen saturation: $99 \%$ in room air. On physical examination, he was in no distress, alert and oriented to time, place and person, normocephalic and atraumatic; and had cranial nerves II to XII intact strength $5 / 5$ in all four extremities and sensations intact, with normal gait. Heart sounds were regular in rate and rhythm with bilateral breath sounds clear and, soft, non-tender abdomen with normal bowel sounds. Peripheral pulses were intact with no peripheral edema noted.

\section{Investigation}

Laboratory evaluation was significant for hemoglobin: $12.3 \mathrm{~g} / \mathrm{dL}$, serum sodium: $140 \mathrm{mmol} / \mathrm{L}$, potassium: $3.9 \mathrm{mmol} / \mathrm{L}$, bicarbonate: $27 \mathrm{mmol} / \mathrm{L}$, blood urea nitrogen (BUN): $95 \mathrm{mg} / \mathrm{dL}$, creatinine: $6.2 \mathrm{mg} / \mathrm{dL}$ (baseline creatinine of $6.0 \mathrm{mg} / \mathrm{dL}$ ), albumin: $4.1 \mathrm{~g} / \mathrm{dL}$, serum glucose: $178 \mathrm{mg} / \mathrm{dL}$ and measured serum osmolality: $317 \mathrm{mosmol} / \mathrm{kg}$. His corrected sodium trend in last six months from current admission was stable compared to serum glucose trend, as illustrated in Fig. 1. CT head showed no acute intracranial abnormality. MRI, as illustrated in Fig. 2, demonstrated a symmetric lesion in the central pons with increased signal intensity on T2-weighted image. MR angiography was unremarkable.

\section{Diagnosis, outcome and follow-up}

After neurological consultation and MRI confirmation, the patient was diagnosed with CPM. In his case, the most

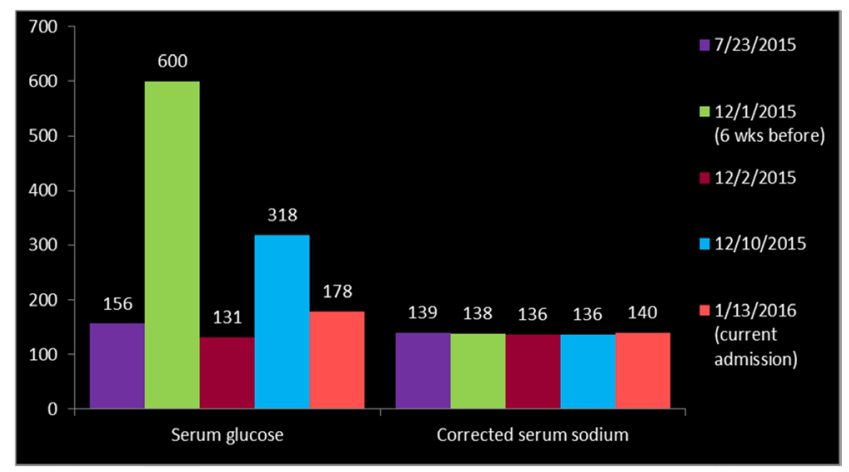

Figure 1

Corrected serum sodium and serum glucose trends.

likely mechanism of CPM could be subacute changes in brain secondary to hyperosmolar hyperglycemia resulting in osmotic demyelination. Despite having stage $5 \mathrm{CKD}$, his electrolytes as well as bicarbonate level were maintained within normal range without requiring any bicarbonate supplementation, thereby CKD is less likely to cause CPM. However, CKD could have been a risk factor for development of CPM secondary to hyperosmolar hyperglycemia. The patient was observed for $48 \mathrm{~h}$ without any change in symptoms or clinical examination. At 2-week follow-up, all symptoms were resolved except for mild persistent gait disturbance. At 8-week follow-up with neurology, the patient was noted to have near-complete recovery.

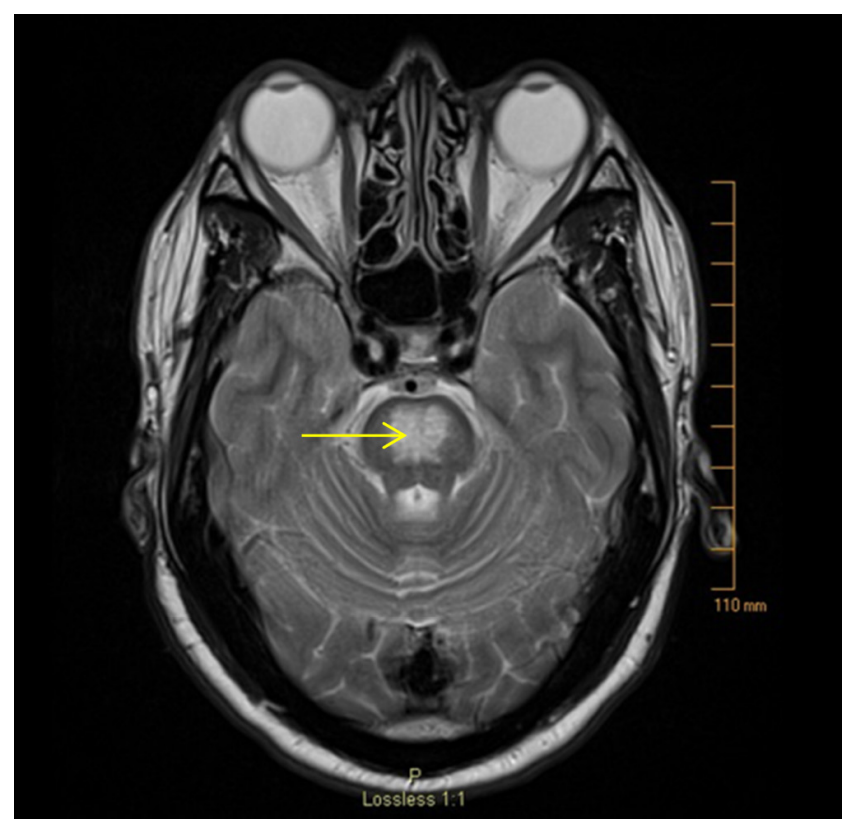

Figure 2

MRI brain T2 image demonstrating a symmetric hyperintense lesion in the central pons. 


\section{Discussion}

Adams et al. first described CPM in 1959 in alcoholics and malnourished patients (1). CPM usually occurs with rapid correction of severe chronic hyponatremia typically with serum sodium below $120 \mathrm{mmol} / \mathrm{L}$. Alcoholism, malnourishment, and severe debilitating illnesses are risk factors for the development of CPM. The composition of the pons with maximum grey and white matter elements makes it more susceptible, however extrapontine involvement is also well described. Some authors prefer the term 'osmotic demyelination' to $\operatorname{CPM}(2,3)$. In chronic hyponatremia, brain cells lose electrolytes into CSF over a few days, rendering the cells isotonic to the extracellular fluid to maintain cell volume by a process called cerebral adaptation. Brain cells, especially oligodendrocytes shrink due to volume loss during rapid correction of tonicity as they cannot synthesize or transport osmoles at such rapid rates, leading to osmotic demyelination (3).

Despite the pronounced fluctuations in serum osmolality, CPM is rarely seen in diabetes (3). To date, there are only few cases of CPM associated with hyperosmolar hyperglycemia in the absence of sodium abnormalities. Most recently in 2015, Saini et al. reported a case of CPM secondary to hyperglycemia, which presented with ataxia and pseudobulbar affect and evolved subacutely over a duration of two weeks (2), similar to the clinical presentation of our patient.

Theories proposed behind the pathogenesis of osmotic demyelination in the setting of hyperosmolar hyperglycemia are: (1) Subacute changes in brain cells secondary to hyperglycemia leading to demyelination (2); (2) rapid changes in osmolality associated with the fluctuations in serum glucose causing osmotic demyelination (4) and (3) hypertonic insult associated with hyperglycemia itself causing demyelination (5). Risk factors for CPM in diabetics include association of diabetic nephropathy requiring dialysis, hepatitis $\mathrm{C}$ and hypertension (6).

A biphasic clinical course is seen in CPM usually, initially encephalopathic phase followed by deterioration later. The clinical manifestations vary based on area of brain involvement and include dysarthria and dysphagia with corticobulbar fiber involvement, flaccid quadriparesis with corticospinal tract involvement and, in severe cases, 'locked-in syndrome.' Tremor, ataxia, movement disorders, and psychiatric and behavioral changes may be seen with extrapontine involvement (3). Rarely CPM can present as isolated gait ataxia (5).
MRI is the imaging technique of choice for diagnosing CPM. Typically, non-contrast enhancing, symmetric hyperintense lesions on T2-weighted images, and hypointense lesions on $\mathrm{T} 1$ weighted images are seen (3).

Treatment of CPM is mainly supportive. Re-lowering the serum sodium may be beneficial if initiated early in CPM caused by rapid correction of severe chronic hyponatremia. Other recommendations including steroids, intravenous immunoglobulin, and thyrotropin releasing hormone lack good evidence (3). There is no specific treatment other than supportive management for non-sodium-dependent osmotic demyelination. The outcome of CPM varies from complete recovery despite initial dramatic presentation to death or permanent disability (3).

Declaration of interest

The authors declare that there is no conflict of interest that could be perceived as prejudicing the impartiality of this research reported.

\section{Funding}

This research did not receive any specific grant from any funding agency in the public, commercial or not-for-profit sector.

\section{Patient consent}

Written informed consent has been obtained from the patient for publication of the submitted article and accompanying images.

Author contribution statement

All the authors were involved in management of this patient during hospital admission. Dr Talluri wrote the case presentation and discussion, and the co-authors formatted manuscript and collected images.

\section{References}

1 Adams RD, Victor M \& Mancall EL 1959 Central pontine myelinolysis: a hitherto undescribed disease occurring in alcoholic and malnourished patients. AMA Archives of Neurology and Psychiatry 81 154-172. (doi:10.1001/archneurpsyc.1959.02340140020004)

2 Saini M, Mamauag MJ \& Singh R 2015 Central pontine myelinolysis: a rare presentation secondary to hyperglycaemia. Singapore Medical Journal 56 e71-e73. (doi:10.11622/smedj.2015065)

3 Martin RJ 2004 Central pontine and extrapontine myelinolysis: the osmotic demyelination syndromes. Journal of Neurology, Neurosurgery, and Psychiatry 75 (Supplement 3) iii22-iii28. (doi:10.1136/ jnnp.2004.045906)

4 Rodríguez-velver KV, Soto-garcia AJ, Zapata-rivera MA, Montes-villarreal J, Villarreal-pérez JZ \& Rodríguez-gutiérrez R 2014 Osmotic demyelination syndrome as the initial manifestation of a hyperosmolar hyperglycemic state. Case Reports in Neurological Medicine 2014 1-5. doi:10.1155/2014/652523 
Endocrinology,

Diabetes \& Metabolism

CASE REPORTS
S Talluri and others

Central pontine myelinolysis

in hyperglycemia
5 Burns JD, Kosa SC \& Wijdicks EF 2009 Central pontine myelinolysis in a patient with hyperosmolar hyperglycemia and consistently normal serum sodium. Neurocritical Care 11 251-254. (doi:10.1007/ s12028-009-9241-9)
6 Ichikawa H, Murakami H, Katoh H, Hieda S \& Kawamura M 2008 Central pontine lesions observed with MRI in four diabetic patients. Internal Medicine 47 1425-1430. (doi:10.2169/ internalmedicine.47.0868)

Received in final form 21 June 2017

Accepted 6 July 2017 